\title{
Nanobodies design for treatment of age-related diseases
}

\author{
Mohammad Mehdi Heidari \\ Department of Biology, Faculty of science, Yazd University, \\ Yazd, Iran \\ heidarimm@yazd.ac.ir
}

\author{
Yuriy L. Orlov \\ Institute of Digital Medicine \\ I.M. Sechenov First Moscow State Medical University \\ Moscow, Russia \\ Novosibirsk State University \\ Novosibirsk, Russia \\ orlov@d-health.institute
}

\begin{abstract}
The problem of the treatment of age related diseases such as Alzheimer disease demands development of new drug design strategies. Reagents that specifically recognize oligomeric morphologies of $\mathbf{A} \beta$ have potential diagnostic and therapeutic value. Nanobodies (Nbs) or Single-domain antibodies are the smallest antigen-binding fragments derived from heavychain-only antibodies. The $\mathrm{E} 1$ nanobody selectively recognizes naturally occurring $A \beta$ aggregates produced in human AD brain tissue We discuss a method for the generation and binding optimization of VHHs that involves the grafting of the complementarity determining regions (CDRs) from already existing, non-camelid antibodies to $\mathrm{VHH}$ frameworks, followed by affinity maturation and target binding improvement using in silico site-directed mutagenesis.
\end{abstract}

Keywords - bioinformatics, nanobodies, protein design, Alzheimer disease, age-related diseases

\section{Motivation and aim}

\section{Motivation}

The prevalence of dementia is increasing in the aging populations at an alarming rate last years. The problem of the treatment of age related diseases including Alzheimer disease demands development of new drug design strategies. Reagents that specifically recognize oligomeric morphologies of $A \beta$ have potential diagnostic and therapeutic value [1].

Aim

Idea is to design in silico nanobodies (Single-domain antibodies) for diagnostics and potential therapy. Nanobodies are special derivatives of antibodies, which consist of only a single chain. The E1 nanobody selectively recognizes naturally occurring $\mathrm{A} \beta$ aggregates produced in human $\mathrm{AD}$ brain tissue indicating that a variety of morphologically distinct $A \beta$ aggregate forms occur naturally.

\section{Methods}

Nanobodies (Nbs) or Single-domain antibodies are the smallest $(12-15 \mathrm{kDa})$ antigen-binding fragments derived from heavy-chain-only antibodies (VHH) [2]. Nanobodies are characterized by high thermal stability and solubility, recognizing uncommon or hidden epitopes of protein targets, unique refolding properties. They exhibit affinities comparable to conventional antibodies and superior tissue penetration.

Nanobodies can also recognize epitopes that remain undetected by conventional antibodies. In addition, nanobodies are shown to be non-immunogenic in humans and are easy to manufacture. Because of these favorable characteristics, nanobodies are suitable candidates for the development of imaging probes, therapeutic agents with neutralizing or receptorligand antagonizing functions, and for targeted drug therapy.

\section{Results and Discussion}

We discuss a method for the generation and binding optimization of VHHs that involves the grafting of the complementarity determining regions (CDRs) from already existing, non-camelid antibodies to VHH frameworks, followed by affinity maturation and target binding improvement using in silico site-directed mutagenesis.

To date, many antibodies and fragments have been generated against various antigens. This existing assortment represents an ideal CDR donor repertoire for our approach. CDR grafting is a powerful technique for transferring binding specificities to other antibody frameworks with desired properties. This is typically done to stabilize or humanize antibodies intended for medical use. To start the mutation process, the CDR, non-CDR loops and the locations on the CDR loops which allow insertions or deletions must be identified. This can be done by aligning nanobody sequences to known structures belonging to the same species. There are publicly available CDR numbering tools that can be applied to a certain sequence. After the identification of the orientation or the sequence of the nanobody, amino acids can be selected for mutation to increase the binding affinity for the antigen.

The rational design of nanobodies based on CDRs derived from conventional, pre-existing antibodies would be of high value to avoid animal immunizations and directly generate binders in the desired framework.

\section{ACKNOWLEDGMENT}

The research was supported by the RSF grant (19-15-00219) and RFBR grant application.

\section{REFERENCES}

[1] Kasturirangan S., Li L., Emadi S., Boddapati S., Schulz P., Sierks M.R. (2012) Nanobody specific for oligomeric ?-amyloid stabilizes nontoxic form. Neurobiol Aging.;33(7):1320-8.

[2] Messer A., Butler D.C. (2020) Optimizing intracellular antibodies (intrabodies/nanobodies) to treat neurodegenerative disorders. Neurobiol Dis. 134:104619. 